\title{
LA PARTÍCULA Y. MARCADOR DISCURSIVO EN EL ESPAÑOL ARGENTINO
}

\author{
The particle $y$. Discursive marker in Argentine Spanish
}

\author{
Cadina M. Palachi* \\ IhuCSo - UNL / UADE \\ cadina.palachi@gmail.com
}

\author{
M. Jimena Morais** \\ UNL / UADER \\ jimena.morais@gmail.com
}

\author{
Palabras clave \\ periferia izquierda; \\ marcadores; \\ discurso; \\ sintaxis
}

\section{RESUMEN}

Nuestro trabajo describe, en el marco de la Gramática Generativa, más específicamente, en el de la teoría cartográfica de la periferia izquierda de la oración (Rizzi, 1997 y siguientes y Cinque, 1999), los valores de la partícula y en el español de Argentina, cuando no es conjunción coordinante. Hemos estudiado un corpus de datos de nuestra variación del español sometido a una encuesta respondida por 125 sujetos de entre 20 y 65 años. Según nuestro análisis, esta partícula -siguiendo la denominación y caracterización de Martín Zorraquino (1992)- en el español de Argentina presenta, además de características propias de la conjunción, las de un marcador del discurso en los términos en que Martín Zorraquino y Portolés Lázaro (1999) lo definen, en tanto una unidad invariable que posee un contenido coincidente con el del discurso y guía inferencias en la comunicación. Revisamos y contrastamos su funcionamiento atendiendo a su alcance, las marcas de polaridad, su no libertad posicional, dentro del Sintagma Complementante, y así observamos tanto un valor enfático (Kany, 1963), como un valor de distanciamiento con respecto al enunciado anterior - contraargumentación o negación- y otro digresor -cambio o inicio de tema nuevo-.

\begin{abstract}
In this paper we describe the values of the particle $y$ in its non-coordinating usages in Argentine Spanish. Our study this particle within the framework of Generative Grammar, more specifically, within the cartographic theory of the left periphery of the sentence (Rizzi, 1997 and following and Cinque, 1999). The corpus of data studied belongs to the variation of Spanish spoken in Argentina and it was submitted to a survey answered by 125 subjects between 20 and 65 years old. According to our analysis, this particle-following the denomination and characterization of Martín Zorraquino (1992)- in Argentine Spanish presents, in addition to the characteristics of the conjunction, those of a discourse marker as Martín Zorraquino and Portolés Lázaro (1999) define it, i.e. it is an invariable unit that has a content coincident with that of discourse and guides inferences in communication. We review and contrast its operation taking into account its scope, the polarity marks, its lack of positional freedom, within the Complementary Phrase, and thus we observe both an emphatic value (Kany, 1969), and a distance value with respect to the previous statement -counter-argument or denial- and another digresor -change or start of a new topic-.
\end{abstract}




\section{A modo de introducción}

\subsection{El problema}

Este trabajo surge a partir de la observación de dos cuestiones: 1. que la conjunción coordinante $y$, en algunos de sus usos, tiene valores que no son propios de la conjunción; es decir que no "sirve para unir palabras o frases” (Moliner, 2007, p. 3085), y 2. que, en los casos en que la partícula aparece con valores no coordinantes se percibe una diferencia semántica entre el mismo enunciado precedido por y y su equivalente sin la partícula.

En el siguiente ejemplo se puede observar que la presencia de $y$ en la respuesta b' no implica coordinación de elementos y, también, que las respuestas b y b' difieren en cuanto a sus significados:

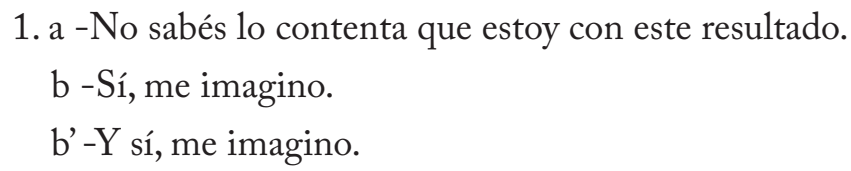

La posición sintáctica de $y$ al comienzo de oración estaría señalando la imposibilidad de coordinación con otro sintagma u oración anterior. Una cuestión diferente es la de responder a la posibilidad de que el enunciado "Y sí, me imagino" esté conectado o coordinado con el enunciado del hablante a. Volveremos sobre este punto, pero consideramos que en este ejemplo $y$ no es un conector discursivo, sino un marcador de modalidad. Este dato fue sometido a una encuesta que respondieron 125 sujetos de entre 20 y 65 años y un 77,6 \% de los encuestados respondió que había una diferencia de significado entre la respuesta de b y la de b'. Un 19,2\% también advirtió una diferencia leve de significado y sólo un 3,2 \% no encontró diferencias entre ambas respuestas. En tal sentido se puede afirmar, tal como hacen Hernanz y Rigau (2006) para referirse a la conjunción que -el elemento que ellas estudian-, que no se trata de un elemento accesorio. Según nuestra interpretación, la diferencia está dada por el énfasis en la afirmación, más marcado en el enunciado de b'. Retomaremos este valor de $y$ en el apartado destinado a la descripción de los datos.

A partir de esta observación, hemos decidido concentrarnos en algunos de los valores semánticos que adquiere la partícula y cuando no funciona como la conjunción copulativa en el español de Argentina. Sin ser exhaustiva, presentaremos una descripción de dichos valores. Nuestra intención es mostrar que $y$, cuando no es una conjunción, se comporta como una partícula ligada al discurso.

Los valores que hemos decidido analizar en este trabajo son los siguientes:

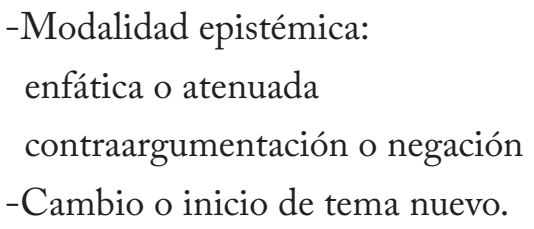

Este elemento, según nuestro análisis, tiene, además de las características propias de la conjunción copulativa, las de un marcador del discurso o, en términos de Biberauer (2008) una "C particle”, es decir, una partícula asociada al sintagma funcional de Complementante en la periferia 
izquierda de la oración y, por lo tanto, ligada al discurso, la interrogación o la modalidad/evidencialidad. Martín Zorraquino y Portolés Lázaro definen los marcadores del discurso como "unidades invariables, (que) no ejercen una función sintáctica en el marco de la predicación oracional -son, pues, elementos marginales- y poseen un contenido coincidente en el discurso: el de guiar (...) las inferencias que se realizan en la comunicación" (1999, p. 4057). Si bien la partícula y no tiene la libertad posicional propia de los marcadores, sí comparte otras características, como señala Camacho (1999, p. 2640) "no coordina sintagmas en sentido estricto puesto que aparece solo uno" de los sintagmas. Por otra parte, es evidente que se trata de una "unidad invariable" en cuanto a su morfología.

También Martín Zorroaquino y Portolés Lázaro se refieren a la capacidad de estos elementos de cambiar de funciones y de valores semánticos, en tal sentido afirman que:

las partículas, es decir, las tradicionales partes invariables del discurso: adverbios, preposiciones y conjunciones, y otros elementos gramaticalizados, pueden desempeñar, en ciertos contextos, funciones que no se ajustan a las que cumplen habitualmente en el marco de la sintaxis oracional. (1999, p. 4055)

Estos elementos adquieren entonces valores de marcadores discursivos. Es también Martín Zorraquino (1992) quien explica que este concepto de partícula es pensado como una categoría específica de "carácter transversal" a esas unidades invariables del discurso y presenta la ventaja de resultar eficiente para describir elementos que operan tanto en la gramática de la oración como en la del discurso.

\section{2. ¿Qué se ha dicho acerca de $y$ ?}

Muchos autores han notado que $y$ tiene otros usos o valores que no son propios de la conjunción coordinante, no sólo en el español de Argentina o Hispanoamérica, sino también en otras regiones de habla hispana y desde tiempos remotos. Sin embargo, algunos de sus usos son característicos sólo del español argentino.

Andrés Bello señala que $y$ "pierde el oficio de conjunción y toma el de simple adverbio en interrogaciones y exclamaciones directas" (1847, p. 391). Entre los ejemplos presentados por Bello se encuentran de Fray Luis de León y de Cervantes, en tal sentido es evidente la antigüedad de este uso no coordinante de la partícula.

Kany trata, entre las conjunciones, la que él llama " $y$ enfática” que no es ligativa y "ocurre en preguntas y exclamaciones" y afirma, siguiendo a Keniston, que "se remonta muy arriba en el curso de la lengua antigua" (1969, p. 464). Los ejemplos que presenta Kany (1969) son interesantes, pues se puede observar que uno de los usos que este autor engloba en la " $y$ enfática" puede ser considerado más como un marcador de cambio de tema o digresión (“¿Y tu padre?”) del que ya hablaremos. Según este autor, existe un uso, diferente al mencionado, que es propio de Argentina y otros países como Chile, Perú y Ecuador, "se la aísla y se la pronuncia con entonación interrogativa, seguida por escrito de puntos suspensivos que indican que la expresión es elíptica” (p. 464). El autor proporciona muchos ejemplos de este valor que considera, siguiendo a Alonso y Henriquez Ureña, "un localismo restringido a la Argentina" (p. 464), aunque indica cautelosamente que este uso también es propio de otras regiones de América. 
El Diccionario de Uso del Español de América y España, Vox, señala que y, registra en el habla coloquial usos no copulativos: "Se utiliza sin valor de enlace para introducir una pregunta sobre el aspecto que se menciona, generalmente las preguntas no llegan a constituir oraciones completas". Y, en otra de las acepciones posibles, indica: "Se utiliza en preguntas y oraciones exclamativas para dar énfasis o indicar disgusto o enfado" (2003, entrada y 2).

María Moliner (2007, p. 3085), en el Diccionario de Uso del Español advierte sobre los valores no coordinantes de esta partícula:

$y$ conj. Sirve para unir palabras o frases en relación de coordinación copulativa (...). A veces, se pone $y$ al principio de una expresión, de modo que aparentemente, no enlaza ésta con nada. Se puede suponer que el enlace se realiza mentalmente con algo pensado o dicho antes o por otra persona. (...). A veces, no es fácil suponer un antecedente tácito y la y debe considerarse como una partícula expletiva (...). Se usa también expletivamente en lenguaje hablado informal entre una interjección y el complemento de ella ‘Caramba y qué niña!'. (La cursiva es nuestra)

Este último ejemplo presentado por Moliner no es propio del español de Argentina, sin embargo, es interesante notar el valor no coordinante de $y$ en otros usos regionales. Hernanz y Rigau (2006) consideran que los usos de estas partículas, aparentemente idiosincrásicos de una región o país, no lo son y tampoco son marginales sino que entran en el rango de microvariación. Estas autoras sostienen, de acuerdo con el marco teórico de la Gramática Generativa (en adelante: GG) y de la cartografía de la periferia izquierda de Rizzi (1997), que existen usos de ciertas conjunciones en construcciones de modalidad interrogativa y exclamativa que, como hemos dicho, no son marginales, sino que se trata de fenómenos de variación dialectal y se repiten en otras variedades alejadas geográficamente.

$\mathrm{Al}$ respecto de $y$, sostienen que si bien otros autores la han considerado marginal -al igual que a la conjunción $0-$, este elemento muestra un valor modalizado y pierde su valor canónico de cópula. Hernanzy Rigau retoman a Bello (1874), quien -como ya hemos dicho-manifestó que en algunos datos (literarios) este elemento $y$ perdía su oficio de conjunción y ganaba uno adverbial en interrogaciones y exclamaciones directas. Las autoras corroboran su perspectiva con muestras del español americano y señalan en particular la variación de Argentina: “(31) a. ¡Y dale con las telenovelas! b. ¡Y pensar que estuve a punto de casarme con él! c. ¿Y me dejáis así, solo y desamparado?”(2006, p. 15). Estas autoras insisten en que, si bien este elemento es considerado por algunos gramáticos como "superfluo", la conjunción $y$ se halla estrechamente relacionada con la fuerza ilocutiva y se genera en la posición de especificador del sintagma fuerza SFuerza.

El Diccionario de partículas discursivas del español compilado por Antonio Briz propone considerar el uso de este elemento $y$ entre signos de interrogación: “¡y...?”. La entrada correspondiente es presentada por María Marta García Negroni y Manuel Libenson, ya que se considera que esta forma es característica de Argentina y México, y se presentan datos recientes (2016-2018). Al respecto se sostiene que $y$ "Presenta el miembro del discurso que introduce como un pedido ya de confirmación, ya de continuación respecto de un discurso o un saber previo implícito que se da como conocido por

1. También observan este comportamiento en el italiano antiguo y moderno y en el catalán. 
locutor e interlocutor" (definición, párr. 1). También observan un valor de pedido de continuación del discurso del interlocutor y otro de atenuación. Acerca de su posición, señalan que siempre inicia el turno de la interlocución -y que también puede ocupar un turno completo-; mientras que, cuando consideran la sintaxis, indican que esta partícula "aparece siempre en estructuras interrogativas o interrogativas-exclamativas, seguidas o no de oraciones interrogativas parciales o totales" (sintaxis, párr. 1).

Como ya se ha señalado en este trabajo, diversos autores (Bello, 1847; Kany, 1969; Hernanz y Rigau, 2006; María Moliner, 2007 y hasta el Diccionario de Vox y del de Partículas discursivas del español) han hecho notar que $y$ no siempre coordina sintagmas o frases.

Escandell Vidal (1999) se refiere al valor no coordinante de $y$ al comienzo de los enunciados interrogativos. La autora diferencia entre la conjunción coordinante y la partícula a partir de la capacidad de una y otra para llevar acento prosódico; "aunque la escritura no refleja este contraste, la partícula $y$ se distingue de la conjunción copulativa $y$ en que esta es siempre átona, mientras que aquella lleva siempre acento prosódico" (p. 3955). Por otra parte, también indica que "las diferencias en el comportamiento discursivo de ambos elementos son muy notables" (p. 3955), puesto que cuando es coordinante la presencia de $y$ está determinada por lo mencionado en el turno anterior, mientras que la partícula, introduce un tema nuevo. Escandell Vidal refiere la introducción de sintagmas nominales (“¿Y Paco?”) y de oraciones condicionales (“¿Y si no congeniamos?”), en nuestra opinión, esta descripción omite la posibilidad de que $y$ también introduzca interrogativas totales que, en el turno del diálogo, marcan el cambio de tema o el comienzo de otro.

Entre los ejemplos recogidos a partir de la encuesta, encontramos una gran cantidad que muestran este valor de $y$ :

\section{2. a. ¿Y al final qué onda con Mati? b. ¿Y, cómo te fue con el médico?}

\subsection{Marco teórico y metodología}

Este trabajo se enmarca en la Gramática Generativa y, consistentemente con la hipótesis de que el lenguaje es de naturaleza biológica y caracteriza a toda la especie humana (es universal), la teoría de la cartografía de la periferia izquierda (Rizzi, 1997 y siguientes; Cinque, 1999 y siguientes) sostiene que los sintagmas a la izquierda de la oración y su ordenamiento jerárquico, generalmente asociados a valores pragmáticos y discursivos, son universales e innatos. La idea de que los sintagmas de la periferia izquierda tienen relación con el discurso, más que con la sintaxis oracional, es discutida en Rizzi \& Bocci (en prensa), quienes sostienen que toda la arquitectura oracional, por encima de los sintagmas funcionales de Concordancia/Tiempo, es sintáctica. En la adquisición de una lengua-I, los sintagmas de la periferia izquierda se activan y están disponibles para los hablantes. En las lenguas-I, algunas de estas categorías no tienen representación morfológica (o fonológica) y no hay partículas que las “encarnen" por lo que pareciera que no están presentes. Teóricamente, se puede postular la existencia, en esos casos, de un morfema 0 (cero). Además, las lenguas y sus variedades pueden presentar diferentes morfemas para cada uno de estos significados o variar en el tiempo. El orden de los sintagmas de la 
periferia izquierda puede variar en las distintas lenguas-I por cuestiones de movimiento. En este marco teórico se analiza la partícula y en la variedad rioplatense del español, de acuerdo con la hipótesis de que la variación intralingüística puede ser reducida a cambios microparamétricos, es decir, los cambios se restringen a los elementos del léxico, de acuerdo con la conjetura Borer-Chomsky.

La teoría generativa ha visto la importancia de estudiar los fenómenos de variación lingüística, y a su vez la incorporación de una perspectiva comparatista ha permitido una renovación en algunos conceptos como el de "construcción gramatical", por ejemplo, según sostienen Hernanz y Rigau (2006). Es así que se observan comparativamente más los fenómenos microparamétricos que las "construcciones", ya que a través del estudio de los primeros se puede llegar a una explicación más profunda de las propiedades gramaticales. Estas autoras sostienen que "Es el estudio comparativo de las pequeñas diferencias entre las lenguas -y no sólo de sus contrastes más llamativos- lo que permite explicar cómo están formadas, hasta qué punto son semejantes y en qué medida pueden divergir" (2006, p. 2). Los estudios microparamétricos han ganado mucho terreno a partir de los aportes del trabajo de Rizzi de 1997 ya mencionado, quien estudió la organización del espacio estructural del SC (Sintagma Complementante), ya que consideró que la "capa” del nudo complementante es más compleja de lo que se consideraba (marcas de subordinación o elementos - qu en función de especificador) y propuso descomponerla en dos niveles - la fuerza (ilocutiva) y la finitud de una oración- los cuales pueden opcionalmente materializarse en otros dos niveles más -Tópico y Foco.

Dentro de las teorías formalistas existe cada vez mayor interés por explicar el cambio de las propiedades gramaticales de las variedades dialectales desde perspectivas teóricas que sostienen que tales cambios son el producto de la competencia entre gramáticas, el reestablecimiento de parámetros o micro-parámetros o del cambio en las propiedades de atracción de las categorías funcionales (Gutiérrez-Rexach y Sessarego, 2014). Una mirada más profunda al estudio comparativo entre lenguas ha permitido observar la necesidad de investigar las variedades de una misma lengua. De manera que la investigación lingüística ha ido enfocando su observación hacia las similitudes y diferencias entre lenguas cercanas o bien entre dialectos o variedades de una misma lengua, especialmente a partir del Programa Minimalista. Señala Kayne (2000) que la pregunta más adecuada en este tipo de investigación es ịcuáles son las unidades mínimas de la variación sintáctica? Este enfoque, que busca encontrar los rasgos mínimos propios de la variedad dentro de una misma lengua, resulta cada vez más necesario para la investigación en lingüística formal y requiere la introducción de conceptos tales como la noción de microvariación morfosintáctica, entendida como las diferencias intralingüísticas que surgen a partir de la interacción de los macroparámetros y los microparámetros.

En este sentido, entendemos que la variación intralingüística también es la que se produce entre la lengua oral y la escrita, por lo que no hablaremos aquí de registro coloquial o formal, ni oral y escrito, ya que en cualquier caso se trata de microvariación, aunque la mayor parte de los datos y ejemplos pertenecen a la oralidad y en un contexto de informalidad.

Para este trabajo hemos utilizado la metodología propia de la investigación en lingüística formal; es decir que, una vez delimitado el problema a partir de observaciones descriptivas guiadas por las hipótesis teóricas, se recogieron muestras. Se diseñó una encuesta online, utilizando un formulario de google, y se consultó a 125 sujetos que realizaron reflexiones conscientes en torno de su conocimiento 
de la lengua, en este caso, de los usos de la partícula y. Luego, se procedió a la descripción y explicación, en términos teóricos, de los problemas planteados. La escritura de este texto surge, entonces, del análisis de los datos a partir de las hipótesis teóricas que enmarcan la investigación. Los datos y ejemplos han sido tomados de diferentes fuentes, especialmente orales, también se han solicitado ejemplos a los sujetos encuestados, todos hablantes de la variedad argentina del español y consultados en el 2020.

\section{4. ¿Es $y$ una partícula? ¿Qué es una partícula?}

La $y$ debe ser catalogada, en su uso más canónico, como una conjunción copulativa puesto que su función gramatical consiste en unir dos sintagmas. Sin embargo, este trabajo se ocupa de otros usos en los que este elemento no liga dos sintagmas, por este motivo, interesa comprender mejor la idea misma de coordinación. José Camacho en la GDLE (1999) entiende que la coordinación se establece entre dos miembros que, tal como indica Bello (1847), son "análogos", sin embargo, hay un problema en establecer si la analogía de los dos miembros es en cuanto a la función sintáctica, a la categoría sintáctica, o si se trata de analogía semántica. Camacho concluye que "se puede reducir el criterio semántico a uno sintáctico. A estos criterios podemos añadir los siguientes: la coordinación debe unir dos o más miembros, y esos miembros deben estar ligados por un nexo" (1999, p. 2637). Desde este punto de vista, la partícula $y$ que nos ocupa presenta una diferencia notable, ya que no establece relación entre dos miembros, en la mayoría de los casos está ubicada al comienzo de una oración o de un enunciado. Por otro lado, la coordinación copulativa es, también según Camacho, "la más neutra en cuanto a su significado básico" (1999, p. 2640). Los posibles significados añadidos a esta conjunción, en opinión de este autor, son temporal, causal y condicional. ${ }^{2}$ En ninguno de los casos que tratamos encontramos estos valores. En tal sentido, entendemos que la partícula estudiada no sería una "conjunción coordinante", debido a que son justamente los diversos significados discursivos y sintácticos que aporta, los que la distinguen de la correspondiente conjunción copulativa.

La discusión sobre la pertenencia de las partículas a una clase diferenciada de otros elementos funcionales no-partículas es extensa. Para este trabajo hemos decidido considerar a $y$ como una partícula relacionada con la periferia izquierda de la oración sin entrar en la discusión acerca de las partículas en sí. Biberauer afirma que

from the perspective of formal considerations like category-type and distribution, then, it is not clear that there is any basis for distinguishing particles from non-particle elements: particles appear to be able to realise the same functional categories than the elements that are not designated particles. (2011, p. 395)

Desde el punto de vista de la GG las partículas pueden ser asociadas a las categorías funcionales, en el caso que nos ocupa, las relacionadas con el dominio de $\mathrm{C}$ (omplementante).

2. No podemos extendernos aquí en revisar la propuesta de Camacho (1999) que puede leerse en la GDLE (1999, p. 2637). 
En opinión de Biberauer (2011) la característica más relevante de un elemento para ser considerado "partícula" es la deficiencia que puede ser morfológica (son elementos indeclinables), fonológica (no reciben acento) o sintáctica (ocupan una posición fija). Si, como indica Escandell Vidal, lo que distingue a la conjunción copulativa de la partícula discursiva, destinada a introducir un tema nuevo en la conversación, es que la segunda lleva acento, entonces la deficiencia fonológica no sería una característica de la y que nos ocupa. Consideramos, en consecuencia, que cumple dos de las tres propiedades para ser considerada "partícula”. Por esta razón continuaremos usando esta denominación que podría ser objeto de revisión en futuros trabajos.

Otra característica presentada por la partícula discursiva $y$ es la que mencionan Bayer Obenauer (2011, p. 450): "It is a general property of discourse particles that they modify the utterance rather than the proposition. They express the speaker's attitude about him-/ herself or about the hearer with respect to the propositional content of the utterance". En este sentido, queda claro que la $y$ es una partícula discursiva.

\section{Descripción y análisis}

\subsection{Dos valores de $y$ que no son “coordinantes"}

Nuestro análisis de los datos nos ha permitido observar que $y$ tiene distintos valores: introduce un cambio de tema, presenta un contraargumento, marca modalidad de manera enfática o atenuada, especialmente cuando va seguida de "bueno".

En este punto nos referiremos a los dos valores que hemos mencionado antes y que repetimos aquí para facilitar la lectura:

- Modalidad epistémica:

enfática o atenuada

contraargumentación o negación

- Cambio o inicio de tema nuevo.

Un uso que no trataremos aquí, pero que interesa mencionar, puesto que ha sido presentado en gran número de los ejemplos aportados por nuestros encuestados, es cuando y encabeza una oración condicional, que debe interpretarse como una invitación, es decir, como una exhortativa:

3. a. ¿Y si vamos a la pile?

b. ¿Y si comemos milanesa?

Interesa mencionar que, a diferencia de otros marcadores del discurso que resultan de procesos de gramaticalización a partir de elementos léxicos, y es originalmente funcional. Las características que diferencian a la partícula de la conjunción son la incapacidad de la primera para ligar o establecer una relación entre dos términos y los matices semánticos que ha adquirido la partícula en relación con las inferencias discursivas. 
Una cuestión central en la consideración de las características de este elemento es la posición que ocupa la partícula en el orden lineal del enunciado; ya que se encuentra siempre a la izquierda, en posición inicial. La conjunción, por su parte, se sitúa entre los dos términos que une; excepcionalmente, puede ir en posición inicial cuando el término anterior se puede reponer, tal como señala Moliner (2007).

Como ya se ha mencionado en relación con el ejemplo (1), la presencia o la ausencia de la partícula en posición inicial de enunciado tiene consecuencias semánticas en la interpretación del mismo, especialmente relacionadas con el valor pragmático del enunciado encabezado por $y$.

En un plano sintáctico, es importante observar que esta partícula encabeza oraciones afirmativas o negativas, interrogativas parciales o totales y exclamativas, aunque, según nuestro análisis, solo cuando va en posición inicial de oraciones enunciativas es un marcador de la modalidad, mientras que en posición inicial de interrogativas es un marcador de cambio de tema o digresión.

Tal como indican Martín Zorroaquino y Portolés Lázaro (1999) entre las características de los marcadores discursivos se encuentra la incapacidad para recibir especificadores o modificadores, así como la imposibilidad de coordinarse entre sí con otros marcadores del discurso. Estos elementos tampoco pueden ser negados. La partícula que analizamos tiene todas estas características.

$$
\begin{aligned}
& \text { 4. -"muy y... } \\
& \text {-*no y... } \\
& \text {-* y de mí... }
\end{aligned}
$$

El comportamiento de los marcadores del discurso es variable en cuanto a la capacidad para aparecer como respuesta independiente. Muchos de estos elementos no pueden ser respuesta en un intercambio de enunciados, sin embargo, otros sí pueden hacerlo. $Y$ tiene esta posibilidad en algunos de sus usos pero no en todos:

5. a- No sabés lo contenta que estoy con la noticia.

$\mathrm{b}^{-*} \mathrm{y}$... (con interpretación “y, sí.")

6. a- Que aburrido es ser un perro. b-Y...

El ejemplo de (6) consideramos que es posible cuando la interpretación supone una negación del enunciado anterior. "Y..." puede interpretarse como "y ... no". Sin embargo, no puede aparecer solo en el caso de (5) donde se interpreta como afirmación enfática.

\subsection{La partícula y marcador discursivo de modalidad epistémica: enfática o atenuada}

La partícula $y$ se comporta en algunos contextos como un marcador de modalidad epistémica afirmando de manera enfática o atenuada un enunciado producido por otro hablante o dentro del propio enunciado. En relación con el ejemplo que en este trabajo se ha presentado como (1), un 64.8\% de los encuestados consideró que la partícula marca un "acuerdo enfático" con el enunciado anterior y un $32 \%$ cree que se trata de una "constatación". Presentamos tres ejemplos en los que se puede apreciar que la partícula manifiesta afirmación, en (7) se puede observar este uso en una narración, 
nos referimos a la expresión "y claro" en la que el entrevistado da la razón a una opinión de otro que menciona inmediatamente después:

7. Le contamos y el tipo preguntó: “¿Y quién va a ser el director?”. "Yo", le dije. Y claro, yo tenía veintiocho años, o veintinueve pero una cara de veinte, más o menos. “¿Usted? No, discúlpeme pero... muy joven”. (Recuperado de CREA, 18/11/2019- ejemplo de prensa 2003)

8. Y sí, era lógico.

9. Y claro, si para eso era...

Cinque define la modalidad epistémica de la siguiente manera: "epistemic modality expresses the speaker's degree of confidence about the truth of the proposition (based on the kind of information she/he has)" (1999, p.86).

Cuando esta partícula tiene valor de marcador de modalidad epistémica siempre encabeza oraciones declarativas: afirmativas o negativas, tal como se puede observar en (7, 8 y 9). Martín Zorroaquino y Portolés Lázaro señalan que hay dos tipos de partículas de modalidad epistémica: las que indican "evidencia" y las que "orientan al interlocutor sobre el origen del mensaje" (1999, p. 4147). Si bien la partícula que nos ocupa pertenece a la primera clase, en este trabajo, sin embargo, hemos decidido no llamarlas "evidenciales", como sí lo hacen los autores mencionados, porque existe una controversia acerca de la definición de marcadores "evidenciales" como "reforzadores de la aserción". Por el contrario, consideramos acertada la idea de "reforzador de la aserción" a la que denominamos "enfática”.

En nuestra opinión la partícula $y$ al comienzo de un enunciado afirmativo, y especialmente cuando va seguida de "sí, claro o bueno", es un marcador de la modalidad epistémica que sirve para corroborar lo dicho por el hablante anterior, ya sea de manera enfática o atenuada. Uno de los encuestados ha sido claro en este sentido al afirmar que usa la expresión "y bueno" "cuando asentís sobre algo que no tiene opciones, que hay que aceptarlo”.

Consideramos que la presencia de la partícula y en el ejemplo (1) permite comprender que el hablante b' no solo afirma lo dicho por el hablante a, sino que expresa también su acuerdo enfático con lo anterior.

Los ejemplos que han aportado los encuestados son:

$$
\begin{aligned}
& \text { 10. a. Y sí, era obvio } \\
& \text { b. Y sí, era lógico. } \\
& \text { c. ¡Y sí, claro! } \\
& \text { d. Y sí, me imagino. }
\end{aligned}
$$

En los ejemplos que presentamos a continuación se puede observar que el hablante manifiesta su acuerdo con un enunciado, aun cuando, probablemente, no esté totalmente de acuerdo con lo afirmado. Sin embargo, la partícula está indicando que, como ha explicitado el encuestado ya mencionado, se trata de "algo que no tiene opciones, que hay que aceptarlo". 
11. a. Y bueno.

b. Y... me parece que...

c. Y sí, si vos decís, así será.

\subsection{Negación o contraargumentación}

De acuerdo con nuestro análisis la misma partícula que introduce una oración aseverativa o enunciativa afirmativa también puede presentarse en posición inicial de una enunciativa negativa para indicar que el hablante no está (del todo) de acuerdo con lo expresado en el enunciado anterior. Se puede observar una escalaridad entre el valor de modalidad epistémica enfática, la modalidad epistémica atenuada (y bueno) y el valor de negación / contraargumentativo en el que el hablante manifiesta su desacuerdo, generalmente, parcial con lo expresado antes:

12. a. Y... me parece que no da.

b. Y... ino sé, eh!

c. Y sí, pero no.

En la encuesta realizada hemos presentado el siguiente diálogo extraído del perfil de Facebook "La gente anda diciendo":

13. -Qué aburrido es ser un perro, ¿no?

-Y... a veces es más aburrido ser humano. (La gente anda diciendo/ chica y chico de unos 20 años, Chacabuco y Rondeau, Córdoba)

La presencia de la partícula no establece una relación de coordinación entre los enunciados, en cambio, marca un distanciamiento con respecto a la opinión vertida por el primer hablante. De hecho, los encuestados respondieron que la partícula "genera una sensación" de distanciamiento (un 50.4\%) y un 29.6\%, especialmente entre los encuestados más jóvenes, sostienen que existe una "contrariedad" en la respuesta. Consideramos que la idea misma de "contrariedad" podría ser revisada, puesto que es probable que los entrevistados hayan considerado que había diferencia de opiniones entre esos hablantes. Es esto, justamente, lo que parece estar indicando la presencia de $y$. Es interesante notar, aunque no tenemos una explicación para esta interpretación, el hecho de que un 34.4\% de los encuestados consideró, además, que se puede reconocer "complicidad entre quienes hablan" a partir de la presencia de la partícula en este caso.

Consideramos que este uso de la partícula tiene un valor contraargumentativo con respecto al enunciado anterior. Incluso podría sobreentenderse una negación (“y... no”), de manera que el enunciado efectivamente es una negación, al menos parcial, del anterior. Sánchez López, en la GDLE (1999), cuando se refiere al "foco de la negación" lo define como "aquel elemento sobre el que recae la exclusión o refutación, de tal manera que puede considerarse responsable de la falsedad o inadecuación de la frase con la realidad" (p. 2577). Es interesante notar que esta autora sostiene que la refutación puede referirse a un elemento efectivamente mencionado o presupuesto y que "a menudo la refutación viene acompañada de un sintagma correctivo que aporta la información que rectifica la del elemento negado" (p. 2577). Una de las maneras de hacer la corrección es justamente, entre otros, "el giro y no". Si bien la autora presenta ejemplos en los que el sintagma introducido por y no refuta una parte del enunciado (ejemplo 43, a. y b. de Sánchez López, 1999, o. 2578 María, y no Luis, habló con Pepe. María 
babló con tus amigos, y no con los míos.), se puede observar que en el ejemplo (13) la partícula y ... indica una corrección del enunciado anterior y podría haberse repuesto el giro “ $y$ no, a veces...”.

Uno de los encuestados nos aporta un ejemplo y realiza una reflexión sobre el mismo: "Si alguien dice 'ese profesor es malísimo' y yo no estoy de acuerdo diría 'y... más o menos”. Es evidente que el uso de la partícula $y$ resulta un marcador discursivo de atenuación, ya que el hablante no quiere presentar una negación tajante, pero rechaza el enunciado anterior. En nuestra opinión este ejemplo muestra que el valor de $y$ contraargumentativo o negador es simplemente una variante del marcador de modalidad epistémica.

En la introducción a esta sección hemos mencionado el hecho de que $y$, cuando se comporta como un marcador que expresa negación, puede aparecer solo. Es interesante observar que esta capacidad se da cuando es respuesta a un enunciado afirmativo o a una pregunta total, no, en cambio, cuando el enunciado anterior es una interrogación parcial, en este sentido podría interpretarse que tiene alcance sobre toda la proposición.

14. - ¿Venís?

$-Y . .$.

15. -Ese profesor es malísimo

$-\mathrm{y} .$.

16. - ¿Cuándo venís? / ¿Quién viene?

$-*$ Y...

\subsection{Conclusiones parciales}

La partícula y en posición inicial con valor de marcador discursivo de modalidad epistémica es compatible con las marcas de la polaridad enfática o no positivas o negativas. Pueden coaparecer con síy también con no pero no es obligatoria la presencia de estos elementos para que la presencia de $y$ permita interpretar al enunciado como una afirmación enfática o atenuada o como una negación, en este último caso, generalmente atenuada.

En relación con los tipos de marcadores relacionados con la negación, Di Tullio y Kornfeld (2013) mencionan los siguientes valores semánticos:

- negación enfática,

- negación polémica, implican una cancelación o frustración de una suposición o expectativa previa,

- desacuerdo relativo, una réplica que no niega el enunciado precedente, sino que indica que no es suficiente.

En nuestra opinión, la partícula $y$ estaría comprendida en el segundo tipo de estos valores "negación polémica".

\subsection{Y cambio de tema}

Otro de los valores semánticos que se puede reconocer en algunos contextos sintácticos es el que ya ha señalado Escandell Vidal (1999), se trata de la introducción de un tema nuevo o de un 
cambio de tema. En este uso la partícula introduce generalmente oraciones interrogativas totales o parciales:

17. -y... al final ¿¿qué te dijo?

18. - ¿Y seguiste yendo al psicólogo?

-Noo. Empecé el gimnasio. Porque los problemas seguían ahí y el culo se me estaba cayendo.

(La gente anda diciendo, mujer de unos 40 años a otra. Colectivo 168. CABA)

En (17 y 18) se trata de un uso digresor, por lo que indica un cambio de tema o sirve para retomar uno que estaba pendiente de otra conversación o de un momento antes en la misma. Escandell Vidal (1999, p. 3955), al referirse a los enunciados interrogativos, indica:

Además, los enunciados interrogativos pueden utilizarse para introducir un tema nuevo, como en el ejemplo de (74): - ¿Y Paco? -Salió a comprar el periódico. Para ello es necesaria la presencia de la partícula $y$, que precisamente marca como tema nuevo al constituyente que le sigue.

Si se observa con atención el ejemplo (74) de Escandell Vidal, se notará que guarda una similitud muy notable con el que Kany (1969) menciona como “ $y$ enfática”. En nuestra opinión, el juicio de Escandell Vidal (1999) es más acertado pues pone en contexto el enunciado como parte de un diálogo.

Uno de nuestros encuestados hace una reflexión interesante al decir "La uso en forma de pregunta, como invitando a la persona a hablar o contar algo de un tema que ya hemos hablado antes. (...) Pero solo oralmente”. De manera que, explícitamente, el hablante reconoce que la utiliza para cambiar de tema o, al menos retomar un tema anterior, pero no inmediato. Ya hemos presentado también un ejemplo en el que la partícula aparece en comienzo de una interrogativa total precediendo a la partícula si con una fuerza exhortativa:

\section{9. - ¿Y si nos tomamos un vino?}

Si bien no presentaremos un análisis de este uso, que retomaremos en futuras investigaciones, consideramos que una hipótesis elegante abogaría por unificar estos dos usos en uno.

Como hemos mencionado, desde la aparición del prestigioso trabajo de Rizzi (1997) se ha prestado mayor atención a la zona de la sintaxis denominada "periferia izquierda" y esto ha permitido observar que la variación intra e interlingüística está, muy frecuentemente, relacionada con la posición que ocupan algunas partículas. ${ }^{3}$ La partícula $y$ en sus usos no coordinantes ha sido considerada propia de algunas regiones de Hispanoamérica, aunque, en línea con las hipótesis de la GG y, tal como señalan Hernanz y Rigau (2006), los fenómenos que se han observado como variaciones idiosincrásicas no lo son y, según se ha podido ver en este trabajo, algunos de los valores que ha adquirido esta partícula en el español de Argentina (tal como el que sirve para introducir un tema) se encuentran en el español de España (según se puede deducir del tratamiento que hace de esta partícula Escandell Vidal y del Diccionario de marcadores discursivos citado más arriba). El valor de marcador de modalidad enfática o

3. Al respecto Rizzi \& Bocci afirman que "Clearly, languages differs as to whether or not, and how, a given position is lexicalized". 
atenuada es más característico de Argentina. En el español de otras regiones hay otras partículas que desempeñan esas funciones, por lo que la variación puede reducirse a una cuestión meramente léxica.

A partir del mencionado trabajo de Rizzi (1997) ha sido evidente que el sintagma que alojaba al complementante (que) no era suficiente para la gran cantidad de elementos que preceden a la oración nuclear. La información que se expresa en la zona propia del SC (Sintagma de Complementante), corresponde a la interfaz entre el contenido proposicional y la estructura superordinada (ya se trate de una cláusula más alta o de la articulación con el discurso). Dicha información es de dos tipos, por un lado, informa acerca del tipo de cláusula (y está relacionada con el Sintagma Fuerza) y por otro lado hay una información que "mira hacia adentro de la cláusula" y tiene relación con el sistema temporal, por lo que se trata de una información contenida en el Sintagma Finitud. De manera que la propuesta de Rizzi (1997) es diferenciar en la zona del SC un SFuerza y un SFinitud, y en estos se insertan morfemas funcionales libres. En un desarrollo más ampliado el autor propone considerar que entre el SFuerza y el SFin se pueden postular dos sintagmas funcionales más: el Sintagma Foco y el Sintagma Tópico. Como se observa, la periferia izquierda de la oración es un espacio en el que se pueden reconocer una gran cantidad de elementos que tienen incidencia en las interpretaciones semánticas de las oraciones.

Cinque (1999) considera que en dicha zona hay una variedad de sintagmas funcionales en los cuales se insertan diferentes tipos de partículas y adverbios. Este autor presenta la modalidad, de una manera muy general, relacionada con "la opinión del hablante o la actitud hacia la proposición". La modalidad expresa la actitud que el hablante tiene hacia la "verdad" del enunciado.

La propuesta presentada en este trabajo sostiene que se pueden reconocer dos valores de la partícula $y$ cuando no es conjunción copulativa:

- marcador de modalidad epistémica

- marcador de cambio de tema (digresor).

La hipótesis, siguiendo a Hernanz y Rigau (2006), es que estos marcadores se sitúan en el especificador del Sintagma de Fuerza que, en la propuesta de Rizzi (1997) es la posición más alta de la estructura oracional. El hecho de que la partícula aparezca siempre en posición inicial y que no sea posible insertar ningún otro elemento antes de la misma es un argumento a favor de tal hipótesis.

\section{Algunas conclusiones e interrogantes}

A partir de la observación de que la partícula $y$, especialmente en el español de Argentina, presenta algunos valores no coordinantes, que en una primera aproximación parecían ser muy diversos, nuestro trabajo ha centrado su atención en observar qué significados aporta la presencia de la partícula a la oración. 
El análisis detenido de los usos ha permitido encontrar los rasgos en común de los, aparentemente, diversos usos y de esta manera nuestra investigación ha intentado unificarlos para lograr una explicación más elegante desde un punto de vista teórico.

Si bien este trabajo es una investigación preliminar, nos ha permitido presentar una descripción de los valores semánticos que aporta la partícula y a los enunciados.

La encuesta que hemos preparado y que han respondido 125 sujetos nos confirmó algunas de las hipótesis preliminares acerca de los diferentes valores semánticos de $y$. Los encuestados no sólo han aportado una gran cantidad de datos y ejemplos, sino que también han realizado reflexiones explícitas acerca de su conocimiento implícito del lenguaje.

La investigación sobre el uso de partículas que operan como marcadores discursivos se ha desarrollado enormemente a partir de las teorías que se enfocan en la pragmática y el análisis del discurso, en cuanto a la lingüística formal, la GG ha puesto su atención en estos elementos desde fines de la década del noventa y ha mostrado un gran desarrollo en este sentido. Por este motivo hemos considerado la importancia de investigar esta partícula desde una perspectiva formalista.

Finalmente, consideramos que este trabajo deja abierto una serie de interrogantes en relación con los usos de la partícula y en las variedades regionales del español. Es importante mencionar que creemos necesario enfocar futuras investigaciones en las variedades cronolectales, puesto que nuestra encuesta ha sido respondida por sujetos cuyas edades oscilan entre los 20 y los 65 años, sin embargo, al analizar los datos no hemos hecho distinciones entre los rangos etarios.

\section{Referencias bibliográficas}

Aliaga Jiménez, J. L.; Almería, L. B. et alii (eds.) (2014). María Antonia Martín Zorraquino. Filología, gramática, discurso. Artículos escogidos [1976-2013]. Zaragoza, Publicación de la Institución Fernando el Católico.

Bayer, J. y Obenauer, H. (2011). Discourse particles, clause structure and question types. The linguistic review, 28, 449-491. https://bit.ly/3emfVqi.

Benincà, P. y Poletto, C. (2004). Topic, Focus, and V2: Defining the CP Sublayers. En Rizzi, L. (ed.), The structure of CP and IP. The Cartography of Syntactic Structures (Vol. 2, pp. 52-75). New York, Oxford University Press.

Biberauer, T. (ed.) (2008). The limits of syntactic variation. Amsterdam, John Benjamins.

Biberauer, T. y Sheehan, M. (2011). Introduction: particles through a modern syntactic lens. The Linguistic Review, 28, 387-410. http://bit.ly/30vLaXJ.

Briz, A. y Grupo Val.Es.Co. Diccionario de particulas discursivas del español. http://bit.ly/2O5LERN. 
Brucart, J. y Hernanz, M. (2012). Las Posiciones Sintácticas. En Gallego, Á. (ed.), Perspectivas de sintaxis formal (pp. 33-110). Madrid, Akal.

Camacho, J. (1999). La coordinación. En Bosque, I. y Demonte, V. (eds.), Gramática Descriptiva de la Lengua Española (pp. 2635-2694). Madrid, Espasa.

Cardinaletti, A. (2011). German and Italian modal particles and clause structure. The Linguistic Review, 28, 493-531. http://bit.ly/3qBBRzY.

Cinque, G. (1999). Adverbs and Functional Heads. A Cross-Linguistic Perspective. New York, Oxford University Press.

Cinque, G. (2004). Issues in Adverbial Syntax. Lingua, 114, 683-710.

Di Tullio, A. y Kornfeld. L. (2013). Marcas de modalidad epistémica en el registro coloquial. En Di Tullio, A. (coord.), El español de la Argentina: estudios gramaticales (pp. 85-105). Buenos Aires, Eudeba.

Escandell Vidal, V. (1999). Los enunciados interrogativos. Aspectos semánticos y pragmáticos. En Bosque, I. y Demonte, V. (eds.), Gramática Descriptiva de la Lengua Española (pp. 3929-3992). Madrid, Espasa.

Gutiérrez-Rexach, J.y Sessarego, S. (2014). Morphosyntactic variation and gender agreement in three Afro-Andean dialects. Lingua 151, 142-161.

Hernanz, M. L.y Rigau, G. (2006). Variación dialectal y periferia izquierda. En Fernández, B. y Laka, I. (eds.), Andolin Gogoan. Essays in honor of professor Eguzkitza (pp. 435-452). Guipúzcoa, Euskal Herriko Unibersitatea.

Kany, C. (1969). Sintaxis Hispanoamericana. Madrid, Gredos.

Kayne, R. (2000). Parameters and Universals. Oxford, Oxford University Press.

Martín Zorraquino, M. A. (1992). Partículas y modalidad. En Holtus, G. et alii (eds.), Lexikon der romanistischen Linguistik (pp. 110-124). Tubinga, Max Niemeyer Verlag.

Martín Zorraquino, M. A. y Portolés Lázaro, J. (1999). Los marcadores del discurso. En Bosque, I. y Demonte, V. (eds.), Gramática Descriptiva de la Lengua Española (pp. 4051-4214). Madrid, Espasa.

Moliner, M. (2007). Diccionario de uso del español. Madrid, Gredos.

Rizzi, L. (1997). The Fine Structure of the Left Periphery. En Haegeman, L. (ed.), Elements of Grammar. Handbook of Generative Syntax (pp. 289-338). Dordrecht, Kluwer Academic Publishers.

Rizzi, L. (2013). The functional structure of the sentence and cartography. En Den Dikken, M. (ed.), The Cambridge Handbook of Generative Syntax (pp. 425-457). Cambridge, Cambridge University Press.

Rizzi, L. y Bocci, G. (en prensa). The Left Periphery of the Clause. En Everaert, M. y van Riemsdijk, H. (eds.), Blackwell Companion to Syntax. II Edition. Blackwell Publishers. 
Rizzi, L.y Cinque, G. (2016). Functional Categories and Syntactic Theory. Annual Reviews, 2, 139-163.

Sánchez López, C. (1999). La negación. En Bosque, I. y Demonte, V. (eds.), Gramática Descriptiva de la Lengua Española (pp. 2561-2634). Madrid, Espasa.

Vox (2003). Diccionario de uso del español de América y España. Barcelona.

*Cadina M. Palachi es Profesora en Letras por la UNL y Doctora en Humanidades y Artes, mención Lingüística, por la UNR. Es Profesora Adjunta a cargo de Latín II, Latín III y Seminario Problemas de Léxico y Morfosintaxis del Español en la FHUC, UNL desde 1998 y Profesora Titular Ordinaria de Lengua Española I en la FHAyCS, UADER. Dirige proyectos de investigación desde 2014. Es directora del Proyecto de investigación CAI+D 2020 Estudios gramaticales del español y lenguas relacionadas: variación lingüistica y problemas de adquisición del lenguaje. Es directora de la Revista Argos, de la Asociación Argentina de Estudios Clásicos.

**M. Jimena Morais es Profesora y Licencia en Letras por la UNL. Es Profesora Asociada a cargo de Latín y cultura latina I y II, Adjunta a cargo de Lengua española II y docente a cargo de Historia de la Lengua en la FHAyCS, UADER, institución en la que ha dirigido adscripciones y un proyecto de investigación. Es Jefa de Trabajos Prácticos de Latín I y II en FHUC, UNL. Fue editora y secretaria de redacción de la revista Argos (2010-2019).

RECIBIDO: 29/11/2020

ACEPTADO: 29/12/2020 